\title{
Crítica de Thomas Reid al sistema de la simpatía de Adam Smith
}

Thomas Reid's Critique of the sympathy system of Adam Smith

MARÍA EltoN

Universidad de los Andes

Instituto de Filosofía

7620001 Las Condes. Santiago de Chile (Chile)

melton@uandes.cl

Abstract: In order to understand Thomas Reid's critique of Adam Smith's Sympathy System it is necessary to determine the relation between this system and the moral phenomenon of sympathy in David Hume. From this perspective, the system can be seen as framed within the 'Theory of Ideas' — as Reid names it - which is developed according to a hypothetical-deductive methodology rather than a Newtonian inductive method, and therefore has no realistic or metaphysical scope.

Keywords: Sympathy, moral faculty, hypothesis, philosophical method.
Resumen: Para comprender la crítica de Thomas Reid al sistema de la simpatía de Adam Smith, es necesario establecer la relación existente entre este sistema y el fenómeno moral de la simpatía en David Hume. Así ese sistema queda enmarcado dentro de la teoría de las ideas, denominada así por Reid, que se desarrollaría según una metodología hipotético-deductiva, y no según el método inductivo newtoniano, por lo que no tendría un alcance realista y metafísico.

Palabras clave: Simpatía, facultad moral, hipótesis, método filosófico. 
^ sí como Hume despertó a Kant de su "sueño dogmático", la lectura cuidadosa que hizo Thomas Reid del Treatise of Human Nature de David Hume le llevó, a través de un largo proceso, a formular su propia teoría del conocimiento humano, por la cual intentó superar el escepticismo de Hume ${ }^{1}$. No es extraño, pues, que Reid haya criticado también el sistema de la simpatía de Adam Smith, en la medida en que este último comparte con Hume la misma estructura fundamental del conocimiento moral.

Sin embargo, así como Reid desarrolló su discusión con la teoría del conocimiento de Hume en una serie de artículos y discursos presentados a la Sociedad Filosófica de Aberdeen, cuyas ideas alcanzaron madurez en su primer libro publicado en 1764, Inquiry into the Human Mind on the Principles of Common Sense ${ }^{2}$, su interpretación del sistema de la simpatía de Smith se encuentra sólo en dos abstracts de Theory of Moral Sentiments, de 2.000 y 750 palabras respectivamente, y en apuntes tomados por sus alumnos de las clases que dictó en la Cátedra de Filosofía Moral de la Universidad de Glasgow, que ocupó durante casi dos décadas, precisamente a partir de la dimisión que hizo de ella Adam Smith ${ }^{3}$. Podemos afirmar, no obstante, con bastante verosimilitud, que la brevedad de esta crítica no se debe a una minusvaloración del pensamiento smithiano, ya que Reid reveló, en varias ocasiones, la alta estima que tenía por su predecesor en la Cátedra que ocupaba. A Lord Kames escribió diciendo que Smith era un hombre genial y un excelente escritor ${ }^{4}$. Existen evidencias de su lectura cuidadosa y atenta de Theory of Moral Sentiments ${ }^{5}$.

1. Cfr. J. Haldane, Thomas Reid: Life and Work, "American Catholic Philosophical Quarterly" LXXIV (2000) 320.

2. Cfr. Ibidem.

3. Cfr. D. F. Norton, J. C. Stewart-Robertson, Thomas Reid and Adam Smith's Theory of Morals, "Journal of the History of Ideas" 41 (1980) 391-392.

4. Cfr. Ibidem, 383. Aunque esta alabanza no tiene ningún tono irónico, Reid apreciaba, por encima de la genialidad, el trabajo atento y paciente, como el que había realizado Isaac Newton. Afirma que este último, al recibir el cumplido de alguien por los adelantos que había hecho en matemáticas y en la filosofía natural, les contestó diciendo que esos descubrimientos se debían más a un trabajo atento y paciente que a otro talento. Cfr. T. REID, Essays on the Active Powers of Man (Edinburgh University Press, Edinburgh, 2010) 61.

5. Cfr. Ibidem, 381. 
Se refería a Smith con un gran respeto, como a su predecesor en la Cátedra que actualmente ocupaba ${ }^{6}$.

A pesar de este aprecio, Reid hizo una crítica al sistema de la simpatía de Smith en los inicios de sus clases en Glasgow. Había desarrollado una aguda y extensa epistemología realista en contraposición a la teoría de las ideas iniciada por Descartes, de la cual, según Reid, Hume había derivado el escepticismo que caracterizó su pensamiento ${ }^{7}$. Desde esta perspectiva, a Reid le preocupaba la impresionabilidad de los estudiantes que tenía al frente, y era reacio a dejar pasar sin crítica ciertos puntos de vista que le parecían perniciosos por sus efectos, al mismo tiempo que era renuente a aparecer como crítico de un filósofo tan eminente y con tanta reputación como Smith, que había enseñado poco tiempo atrás al mismo auditorio al que se dirigía ahora ${ }^{8}$.

En mi opinión, la interpretación crítica que hace Reid del sistema de la simpatía de Smith no tiene sólo un valor histórico filosófico, sino también un valor para la filosofía práctica actual, dentro de la cual una cuestión principal es cómo llegamos a hacer juicios morales que dirijan nuestras acciones. Desde la is-ought question planteada por Hume, el pensamiento filosófico acerca de si es posible hacer juicios racionales acerca de las acciones morales ha derivado, en general, en conclusiones negativas, especialmente en el campo de la filosofía analítica, uno de cuyos mayores representantes es Ayer, quien ha afirmado definitivamente el emotivismo moral, negando radicalmente la posibilidad de un cognotivismo ético ${ }^{9}$. La discusión acerca de la crítica que suscitó el emotivismo moral en su misma época, da luces nuevas acerca de ciertos elementos que nos pueden permitir salir del impasse en que se encuentra hoy dicho cognitivismo.

6. Cfr. Ibidem.

7. Cfr. Manuscript, en T. REID, An Inquiry Into The Human Mind On the Principles Of Common Sense (The Pennsylvania State University Press, Pennsylvania, 1997) 290.

8. Cfr. D. F. Norton, J. C. Stewart-Robertson, op. cit., 381.

9. Cfr. C. I. MAssini, La falacia de la "falacia naturalista", "Persona y Derecho" 29 (1993) 47-95. 
Por otra parte, la investigación acerca de la índole del juicio moral simpatético, expuesto por Smith en su Theory of Moral Sentiments, ha cobrado una especial relevancia en los últimos años, dando lugar a variadas interpretaciones entre los estudiosos, entre las que cabe destacar la inclusión que ha hecho Tugendhat de esa teoría en uno de los capítulos de sus Lecciones de Ética ${ }^{10}$. No se puede, por tanto, pasar por alto, la breve, pero sustanciosa interpretación que hizo Reid, contemporáneo de Smith, acerca de la aprobación moral simpatética. Sin embargo no se ha profundizado en ella. Su estudio ha sido comenzado por H. Duncan y Robert M. Baird ${ }^{11}$, D. F. Norton y J. C. Stewart-Robertson ${ }^{12}$, y J. Reeder ${ }^{13}$, a quienes les debemos habernos dado a conocer los manuscritos originales donde se encuentra. Pero es una discusión susceptible de una interpretación más honda, la cual pretendo incoar en este artículo, tratando sólo un aspecto de ella, que podemos formular así: ¿se puede hacer un juicio verdadero acerca del valor moral de nuestras acciones a partir de la simpatía, o esta es un sentimiento que debe subordinarse a una facultad moral racional?

Ya que Hume fue el primero en proponer una teoría de la simpatía para explicar el juicio moral, y debido al aprecio que Smith tuvo por su Treatise of Human Nature ${ }^{14}$, en la primera sección de este artículo, después de exponer la breve crítica de Reid a Smith sobre la simpatía, me referiré a la fundamentación de la aprobación moral simpatética smithiana en la teoría de la simpatía de Hume, a pesar de cierta diferencia que el mismo Smith establece con ella. La comprensión de los presupuestos humeanos es clave para entender la crítica reidiana a Smith. Explicaré también por qué se puede afirmar que Hume y Smith comparten una misma metodología para el trata-

10. Cfr. E. Tugendhart, Lecciones de Ética (Gedisa, Barcelona, 1997) 273-299.

11. Cfr. E. H. Duncan, Thomas Reid on Adam Smith's Theory of Morals, "Journal of the History of Ideas" 38 (1977) 509-522.

12. Cfr. D. F. Norton, J. C. Stewart-Robertson, op. cit., 381-398; y J. C. Stewart-Robertson, D. F. Norton, Thomas Reid on Adam Smith's Theory of Morals, "Journal of the History of Ideas" 45 (1984) 309-321.

13. J. REEDER, On Moral Sentiments (Thoemmes Press, Bristol, 1997) 69-89.

14. Cfr. J. RAE, Life of Adam Smith (London Elibron Classics, London, 2005) 15-16, 24. 
miento de la simpatía, la cual es cuestionada por Reid. En la segunda sección abordaré el tratamiento que hace Reid de la subordinación de la aprobación simpatética al juicio racional de una facultad moral original, la existencia de la cual es demostrada por este autor a partir de la metodología filosófica newtoniana.

El fundamento de la filosofía reidiana es un naturalismo providencialista basado en la metodología científica de Newton, por medio del cual Reid sostiene que tenemos una facultad moral original que nos permite hacer juicios morales verdaderos, previos al sentimiento de simpatía ${ }^{15}$. Esos juicios son del sentido común, entendido no como una opinión mayoritaria, sino como una facultad, según veremos. Newton fue, sin duda, un referente principal del contexto intelectual de Reid, quien, sin embargo, ha sido considerado como quien tuvo la comprensión más profunda de la ciencia newtoniana entre los filósofos escoceses del siglo XVIII ${ }^{16}$. Esto es manifiesto en las frecuentes referencias a las reglas del filosofar de Newton que encontramos en sus obras ${ }^{17}$. Asumidas con rigor por Reid, estas reglas constituyen la clave para entender la crítica que hace al sistema de la simpatía de Smith, el cual no las habría seguido a pesar de su admiración por la ciencia experimental newtoniana.

15. Reid fue introducido en un naturalismo providencialista y, por tanto, en la aplicación del método científico de Newton a la filosofía moral, por George Turnbull, su maestro de juventud. Cfr. D. F. NorTOn, George Turnbull and the Furniture of the Mind, "Journal of the History of Ideas" 36/ 4 (1975) 712-714. Bajo el título de su obra principal, Principles of Moral and Christian Philosophy (Liberty Fund, Indianapolis, 2005), Turnbull cita la siguiente afirmación de Newton: "And if NATURAL PHILOSOPHY, in all its Parts, by persuing this Method, shall at length be perfected, the Bounds of MORAL PHILOSOPHY will also be enlarged". Cfr. I. NEWTON, Mathematical Principles of Natural Philosophy and Optics (Encyclopedia Britannica, Chicago, 1971) 543.

16. Cfr. A. Broadie, Reid in context, en T. Cuneo, R. van Wondenberg (eds.), The Cambridge Companion to Thomas Reid (Cambridge University Press, New York, 2004) 40; y W. J. Ellos, Thomas Reid's Newtonian Realism (University Press of America, Washington, D.C., 1981) 22ss.

17. En efecto, en An Inquiry cit., encontramos cuatro referencias al método inductivo de Newton, en Essays on the Intellectual Powers (The Pennsylvania State University Press, University Park, Pennsylvania, 2002) hallamos seis y en Essays on Active cit. se encuentran dos. 


\section{LA SIMPATÍA SMITHIANA EXPLICADA A PARTIR DE LA TEORÍA HUMEANA DE LA SIMPATÍA. MÉTODO HIPOTÉTICO}

Según Reid, Smith intenta en su sistema reducir la moralidad a unos pocos principios originales, porque todos nuestros sentimientos morales se resuelven en la simpatía, la cual, a su vez, se resuelve en el amor a sí mismo, que recibe un cambio de dirección por la operación de la imaginación ${ }^{18}$. Nos centraremos en este artículo en dos elementos de esa interpretación: 1) la simpatía smithiana es un sentimiento que nos permite hacer aprobaciones morales; y 2) la simpatía surge a partir de una operación de la imaginación. Para descubrir cual es el método implícito en la explicación que hace Smith de esos elementos de la simpatía, es necesario precisar en que consiste la influencia de Hume en su teoría, y porqué Reid afirma que ambos utilizan un método hipotético-deductivo para el desarrollo de su filosofía moral.

\section{a) Simpatía espontánea y simpatía técnica. Influencia de Hume en Smith}

Haciendo una síntesis del sistema de la simpatía smithiano, Reid explica que la simpatía es suscitada cuando nos ponemos a nosotros mismos, por medio de la imaginación, en la situación de otra persona, y desde allí formamos una idea de sus sentimientos, e incluso sentimos algo que, aunque en un grado más débil, es semejante a esos sentimientos, simpatizando o no con ellos ${ }^{19}$. El sentimiento de simpatía es, pues, consecuencia de una operación de la imaginación ${ }^{20}$, y lleva consigo, cuando se produce, una aprobación moral. La simpatía es así, para Smith, un término técnico ${ }^{21}$.

18. Cfr. Birkwood Collection, MS.2131/2/II/6, en J. C. STewart-RoberTson, D. F. NORTON, op.cit., 311.

19. Cfr. Ibidem; y A. SMITH, Theory of Moral Sentiments (Liberty Classics, Indianapolis, 1976) I.I.1.

20. Cfr. Birkwood Collection, MS.2131/2/II/6, en J. C. Stewart-RoberTson, D. F. NORTON, op.cit., 311.

21. Cfr. A. Broadie, The Scottish Enlightenment: an Anthology (Cannogate Classics, Edinburgh, 1997) 155. 
Pues bien, esa explicación de la causa de la simpatía, según Reid, atenta tanto contra la naturaleza de ese sentimiento, como también contra una concepción adecuada de las operaciones de la imaginación. En efecto, cuando me imagino o me concibo en la condición de una persona que sufre, puede ser natural que, como consecuencia, me imagine sufriendo lo que padece, pero también me puedo imaginar todo eso sin sufrir en absoluto. Imaginar un dolor y sentir un dolor son dos cosas completamente distintas, y la primera operación no lleva consigo necesariamente la segunda. La mera imaginación de un padecimiento que yo pueda tener, no es padecer, como la imaginación de estar en Francia no es estar en Francia $^{22}$. Cuando siento simpatía por una persona, por otra parte, continúa Reid, no soy consciente de un cambio de persona. Hay muchas operaciones de la mente que escapan a nuestra advertencia, por falta de una reflexión atenta, pero no podemos suponer unas operaciones de la imaginación sin ser conscientes de ellas ${ }^{23}$.

Reid acusa a Smith de inventar una hipótesis para explicar este fenómeno moral, lo cual constituye un método filosófico que atenta contra las reglas del filosofar de Newton ${ }^{24}$. La primera de esas reglas establece que no se deben admitir otras causas de los efectos naturales que aquellas que sean tanto verdaderas como suficientes para explicar esas apariencias ${ }^{25}$. Esta regla es aplicable, según Reid, a las causas de los efectos naturales, sean estos relativos a la materia o a la mente ${ }^{26}$. Recordemos que, para Newton, el método de la filosofía natural serviría, en el futuro, para perfeccionar la filosofía moral ${ }^{27}$.

Afirma también Newton que cualquier cosa que no sea conocida a partir de los fenómenos por inducción, es una hipótesis; y las hipótesis, ya sean metafísicas o físicas, o basadas en cualidades

22. Cfr. Birkwood Collection, M.S. 2131/2/II/6, en J. C. STEWART-RoberTson, D. F. NORTON, op. cit., 311.

23. Cfr. Ibidem.

24. Cfr. Ibidem.

25. Cfr. I. NeWTON, op. cit., 270. Reid destaca la importancia de la aplicación de esa regla al trabajo filosófico en T. REID, Essays on Intellectual cit., 51.

26. Cfr. T. REID, Essays on Intellectual cit., 51.

27. Cfr. nota 15 . 
ocultas, o mecánicas, no tienen lugar en la filosofía experimental ${ }^{28}$. Según este presupuesto, no se debe dar lugar, según Reid, a conjeturas o hipótesis en filosofía, y se debe poner a prueba cualquier opinión por la piedra de toque de los hechos y la experiencia. Lo que puede ser conocido a partir de los hechos debidamente observados, o suficientemente atestiguados, es la voz de Dios, y no una ficción de la imaginación humana ${ }^{29}$.

Un modo principal y directo de observación de nuestros fenómenos mentales es "la reflexión atenta sobre las operaciones de nuestra mente" ${ }^{30}$. La manera de conocer las obras de la naturaleza, accesible a todos los seres humanos, es la observación, según una tendencia de nuestra constitución, a partir de la cual establecemos leyes generales. Así Newton descubrió la ley de la gravitación y de las propiedades de la luz. Las reglas del filosofar de Newton son máximas del sentido común, y se practican en la vida ordinaria ${ }^{31}$. La teoría de la simpatía de Smith debería ser admitida sólo si puede ser conocida por cualquiera que sea capaz de una reflexión cierta sobre sus propios sentimientos, en cuyo caso sería verdadera ${ }^{32}$.

Pues bien, por reflexión atenta sobre nuestros sentimientos, Reid considera que la simpatía es un instinto natural propio de nuestra constitución, involuntario, que se manifiesta como un sentimiento social que nos inclina a aliviarle el dolor al otro, el cual no es virtuoso en cuanto tal, por lo que se puede encontrar en los malvados, por ejemplo en una madre que está dando muerte a su hijo. Aunque hay que admitir la existencia de una simpatía virtuosa, pero que es propia de una determinación de la voluntad, la cual supone una facultad moral capaz de percibir lo bueno y lo malo ${ }^{33}$. Además,

28. Cfr. I. NEWTON, op. cit., 371. Reid adopta esta afirmación newtoniana en T. REID, Essays on Intellectual cit., 52.

29. Cfr. T. REID, Essays on Intellectual cit., 51.

30. Cfr. T. REID, Essays on Intellectual cit., 56

31. Cfr. T. REID, An Inquiry cit, 11-12.

32. Cfr. Cfr. Birkwood Collection, MS.2131/2/II/6, en J. C. StewarT-RoberTson, D. F. NORTON, op.cit., 311.

33. Cfr. T. REID, Essays on the Active cit., 46-51. En este capítulo Reid explica la mutua influencia entre motivos y voluntad, tema en el que no podemos entrar en este artículo por falta de espacio. 
simpatizamos más con los que queremos y estimamos, y con los que estamos conectados, y en relación con una persona, simpatizamos más con sus penas que con sus alegrías. Por otra parte, simpatizamos más con los inocentes que con los culpables. En cuanto a un cambio de persona con el otro, nos ponemos más en la persona de un amigo, de una relación, de una persona inocente, que en la persona de un extraño, de un enemigo o de un culpable. En todos estos casos la simpatía es previa al cambio de persona, y no una consecuencia de esta operación en la imaginación ${ }^{34}$.

¿Cual es la hipótesis de Smith, en términos reidianos, según la cual la simpatía se transforma en un término técnico, dejando de ser un mero sentimiento espontáneo? No es explicada en los apuntes de clases en que se conserva la crítica de Reid a la simpatía smithiana. Suponemos que en esas clases él explicaba su propia filosofía, y se refirió a Smith tangencialmente.

Pero nosotros podemos reconstruir esa hipótesis remontándonos al origen del sentido técnico de la simpatía smithiana, que se encuentra en Hume, el cual entiende este fenómeno como una asociación de ideas e impresiones en la imaginación; y podemos analizar críticamente esa hipótesis desde la interpretación que hace Reid de esa teoría asociacionista en sus obras, la cual es atribuida por Reid a Hobbes, quien la habría sugerido, y a Hume, quien la habría argumentado con claridad ${ }^{35}$. La comprensión de la hipótesis smithiana de la simpatía depende, por tanto, de su afinidad con la simpatía humeana.

Según Haakonssen, Hume desarrolló en el Treatise una teoría de la imaginación, la cual fue adoptada por Smith como la médula de su filosofía de la mente en Theory of Moral Sentiments. Tanto para Hume como para Smith, la imaginación es una facultad mental por medio de la cual las personas crean una esfera humana dentro del mundo natural ${ }^{36}$. La creación de esa esfera humana se realiza princi-

34. Cfr. Birkwood Collection, MS.2131/2/II/6, en J. C. STEWART-RoberTson, D. F. NORTON, op. cit.,312.

35. Cfr. T. REID, Essays on Intellectual cit., 347-348.

36. Cfr. K. HaAkonssen, Introduction to The Theory of Moral Sentiments (Cambridge University Press, Cambridge, 2002) xii-xiii. 
palmente por medio del fenómeno de la simpatía, el cual es definido por Hume como la "conversión de una idea en impresión por medio de la fuerza de la imaginación" 37 .

Hume explicó este fenómeno moral ${ }^{38}$, con base en su teoría acerca del origen, composición, conexión y abstracción de las ideas ${ }^{39}$. En efecto, al comienzo del Treatise, Hume establece ciertos principios de los cuales se deriva todo su sistema, tanto del entendimiento, como de las pasiones y de la moral. Afirma que todas las percepciones de la mente humana se reducen a impresiones e ideas, y que la diferencia entre ambas consiste en el grado de fuerza y vivacidad con que inciden en la imaginación. Las impresiones entran con fuerza y violencia, y las ideas son imágenes débiles de las impresiones ${ }^{40}$. Las impresiones pueden ser, a su vez, de dos clases: de sensación, que surgen originariamente en el alma por causas desconocidas, y de reflexión, que se derivan de nuestras ideas. Las segundas constituyen en la mente una serie de pasiones o sentimientos, las cuales son copiadas, a su vez, por la imaginación y la memoria, convirtiéndose en ideas ${ }^{41}$. Las ideas se conectan en la mente por ciertas leyes de asociación, a partir de las cuales se desarrolla el pensamiento, por una parte, y surgen las pasiones y aprobaciones morales, por otra ${ }^{42}$.

Pues bien, Reid considera que ese sistema filosófico es hipotético, porque es deducido a partir de unos pocos principios - unas impresiones e ideas originales y tres leyes de asociación ${ }^{43}$ - , cuya existencia no es demostrada por la experiencia. El sistema no es, pues, ni científico, ni filosófico. Cualquier sistema construido en base a conjeturas es denominado ciencia de manera impropia, porque las conjeturas dan lugar a opiniones, pero no a ciencia ${ }^{44}$. En uno de sus discursos a la Aberdeen Philosophical Society, antes de

37. Cfr. D. Hume, A Treatise of Human Nature (Clarendon Press, Oxford, 1960) III. VI.

38. Cfr. Ibidem, II.I.XI.

39. Cfr. Ibidem, II.I.XI.

40. Cfr. Ibidem, I.I.I.

41. Cfr. Ibidem, I.I.II.

42. Cfr. Ibidem, I.I.III.

43. Cfr. T. REID, An Inquiry cit., 22.

44. Cfr. T. REID, Essays on Intellectual cit., 47. 
escribir An Inquiry, Reid examina la teoría de las ideas iniciada por Descartes considerando que Hume sacó las últimas consecuencias de esa teoría al afirmar que el objeto propio del pensamiento humano son las impresiones e ideas que el hombre encuentra en la memoria e imaginación, de cuyo origen en la mente no dio ninguna fundamentación ${ }^{45}$.

\section{b) Adopción del método bipotético por Smith}

Smith adoptó la estructura y funcionamiento de la mente humana establecida por Hume ${ }^{46}$, lo que nos permite interpretar su sistema de la simpatía a la luz de la explicación que hace Hume de la simpatía en el Treatise. Esto es así a pesar de las diferencias existentes entre ambos. En efecto, Hume construyó una teoría abstracta de la simpatía, con su propio lenguaje, y trató de introducir las experiencias comunes a todos los hombres y sus expresiones linguísticas dentro de esa teoría. Smith, por el contrario, intentó acomodar esa teoría abstracta dentro de la estructura conceptual de la experiencia y del lenguaje ordinario ${ }^{47}$. Por eso, la teoría de la mente que está en la base del proceso de la simpatía se encuentra claramente explícita en el Treatise de Hume; mientras que en Theory of Moral Sentiments está sólo implícita. Por otra parte Smith, sin apartarse de esa estructura de la mente, introduce una nueva causa de la simpatía, como veremos, haciendo de ella un fenómeno más complejo y sofisticado que la simpatía humeana.

Aunque Smith explique la teoría de la simpatía por conceptos experienciales propios del lenguaje ordinario, no lo hace de acuerdo a la primera regla del filosofar de Newton, que exige explicar las causas de los fenómenos por inducción a partir de la observación

45. Cfr. T. REID, An Inquiry cit., 290.

46. Cfr. A. SмIтH, Principles which lead and direct Philosophical Inquiries; illustrated by the History of Astronomy, en Essays on Philosophical Subjects (Liberty Classics, Indianapolis, 1980) 33-53; y A. SMITH, Lectures on Rhetoric and Belles Lettres (Liberty Classics, Indianapolis, 1983) 203ss.

47. Cfr. K. HaAkonssen, The Science of a Legislator (Cambridge University Press, New York, 1981) 45. 
de ellos mismos — regla adoptada por Reid como el método filosófico por excelencia-, y no por hipótesis o conjeturas. Tanto Hume como Smith eran admiradores de Newton. Hume profesa en la introducción al Treatise, su intención de desarrollar una ciencia del hombre según el método de Bacon y Newton ${ }^{48}$. Sin embargo adopta una mecanicismo de tipo cartesiano al explicar los fenómenos de la mente, enteramente alejado del método de análisis newtoniano ${ }^{49}$. En cuanto Smith adopta la estructura de la mente establecida por Hume, ese mecanicismo fundado en una hipótesis está implícito en el juicio simpatético propio de su antropología.

Smith también manifiesta, en diversas ocasiones, un gran aprecio por Newton. Sin embargo, su única referencia a la metodología newtoniana se encuentra en Lectures on Rhetoric, donde, al manifestar su preferencia por la metodología newtoniana, explica su parte compositiva o axiomático-deductiva, sin referencia a su método de análisis, filosófico y realista, propuesto por Reid para hacer una filosofía de la mente $^{50}$. En Theory of Moral Sentiments elogia a Newton una vez, por

48. Cfr. D. Hume, Treatise cit., xxi.

49. Newton afirma en Optics, que la investigación de cosas difíciles debe hacerse por el método del análisis, que debe preceder al método de la composición. El análisis consiste en hacer experimentos y observaciones, sacando conclusiones generales por medio de la inducción. No se deben admitir hipótesis metafísicas y físicas en el método experimental (Cfr. NEWTON, op. cit., 543). Rechaza el mecanicismo cartesiano, el cual parte de la hipótesis de un mundo puramente corporal, enteramente determinado por leyes mecánicas supuestas por la imaginación. Aunque a veces adopta un tono hipotético respecto a fenómenos particulares, Newton no lo hace declarativamente, ni constituyendo un sistema cerrado como el cartesiano. Cfr. A. Gabbey, Newton, Active Power, and the Mecanical Philosophy, y H. Stein, Newton Metaphysics, ambos en B. Cohen, G. E. Smith (eds.), The Cambridge Companion to Newton (Cambridge University Press, Cambridge, 2002) 335-43 y 282ss. Otras interpretaciones en este mismo sentido se encuentran en A. JaNIAK, Isaac Newton, en The Oxford Handbook of British Philosophy in the Seventeenth Century (Oxford University Press, Oxford, 2013) 96ss; A. E. SHAPIRO, Newton's "Experimental Philosophy", "Early Science and Medicine" 9/3 (1884) 185217; B. CoHEn, Hypotheses in Newton's Philosophy, "Physis, Rivista Internazionale di Storia della Scienza 8 (1966) 163-184. Según Schliesser, existe una similitud fundamental entre la asociación de ideas y sus causas en la filosofía de la mente de Hume, y el mecanicismo cartesiano anterior a Newton. Cfr. E. SCHLIEsSER, Two Definitions Of 'Cause', Newton, and The Significance Of The Humean Distinction Between Natural and Philosophicals Relations, "The Journal of Scottish Philosophy" 5/1 (2007) 86-9.

50. Cfr. A. Smith, Lectures on Rhetoric cit., 145-146. Como vimos anteriormente, 
ser un gran matemático, pero no se refiere a su metodología ${ }^{51}$. En History of Astronomy dedica varias páginas a sus descubrimientos sobre el sistema planetario, alabándolo por su genio superior y su sagacidad, como por la prevalencia que tendría en el tiempo su sistema, pero sin referirse a su metodología ${ }^{52}$. No encontramos, por tanto, ninguna intención en Smith de atenerse al método de análisis newtoniano, pero sí cierta admiración por la parte menos filosófica de esa metodología, la axiomático-deductiva. Por otra parte, fundamenta su teoría del conocimiento desarrollada en History of Astronomy en una asociación de ideas en la imaginación de tipo humeana ${ }^{53}$, la cual es posible rastrear también en Theory of Moral Sentiments.

Antes de hacer esta búsqueda, veamos primero, en síntesis, qué dice Hume sobre la simpatía ${ }^{54}$. Considera que nuestra virtud no es tal si no está secundada por la opinión y sentimientos de los demás, lo que se produce a través de la simpatía, la cual nos inclina a recibir por comunicación las inclinaciones y sentimientos de los otros. Conocemos esas afecciones por sus efectos en su semblante y en su conversación, lo que se manifiesta en una idea, la cual tiene tal fuerza y vivacidad en la mente del observador, que se convierte en una impresión y llega a ser una pasión que produce una emoción igual a la afección original, espontáneamente. Cualquier principio o pasión en otro se encuentra en cierto grado en nosotros mismos, debido a la semejanza existente por naturaleza en todas las creaturas humanas. Esta semejanza contribuye a que penetremos en los sentimientos de los otros, adoptándolos con facilidad y placer, lo cual facilita la simpatía, porque la imaginación transmite vivacidad a la idea con mayor facilidad. Esta transmisión se realiza también porque

Newton afirma que el método de análisis debe preceder al método de síntesis o compositivo. Por medio del primero se descubren las causas, y se establecen principios que son posteriormente asumidos por el segundo, para explicar los fenómenos que proceden de ellos (Cfr. NEWTON, op. cit., 543).

51. Cfr. A. SMITH, Theory of cit., III.2.20

52. Cfr. A. SMith, Principles which lead cit., 97-105.

53. Cfr. ibidem, 37-53. Un estudio de esa teoría se encuentra en R. LÁZARO, La sociedad comercial en Adam Smith. Método, moral, religión (Eunsa, Pamplona, 2002) 111-122. Lázaro afirma que la influencia de Hume en Smith es clara en cuanto al papel que Smith hace jugar a la imaginación en el conocimiento.

54. Cfr. D. Hume, Treatise cit., 316-320. 
cualquier objeto que se relacione con nosotros mismos, es decir con el yo, tiene gran vivacidad. Tenemos siempre íntimamente presente la idea o impresión de nosotros mismos, o del yo, que es una concepción muy viva de nuestra propia persona. Esa relación de semejanza se refuerza con otras relaciones que la acompañan, como es la de contigüidad, de la sangre, del conocimiento, etc. El fenómeno de la simpatía se produce, pues, según Hume, por una asociación de ideas e impresiones dentro de la imaginación, según los principios que él establece en el Libro I del Treatise, como vimos. La simpatía es, pues, un fenómeno mecánico asociativo.

Smith desarrolla más y sofistica ese mismo mecanismo, sin explicar, sin embargo, la teoría de la mente subyacente. Le parece que los filósofos, en los últimos años han considerado sólo la tendencia de los afectos, y han puesto poca atención en las causas que excitan esas afecciones. En la vida ordinaria, en cambio, cuando uno juzga la conducta de una persona y los sentimientos que la dirigen, tiene en cuenta siempre ambos aspectos ${ }^{55}$. Entre los factores que son causa de la reacción simpatética del espectador, Smith incluye la situación en que se produjo la pasión original ${ }^{56}$, constituyendo a las circunstancias en la causa de la pasión ${ }^{57}$, lo que le permite distinguir entre el objeto de la simpatía, que es la pasión del otro, y su causa, que es la situación completa que la origina. "El mérito de su favorito" dice, recurriendo a una experiencia ordinaria, "no es tan grande, su desgracia no es tan terrible, su provocación no es tan extraordinaria, como para justificar una pasión tan violenta" ${ }^{58}$.

Para Smith, la causa de la simpatía establecida por Hume, es decir la percepción de la expresión de una pasión en el otro, es insuficiente para provocar una evaluación moral simpatética. Hume sólo considera los motivos que originan una acción, los cuales son juzgados moralmente por una aprobación implícita en el sentimiento de simpatía. Smith, en cambio, considera esos motivos como una reacción conveniente o no a las circunstancias que los originaron. Así

55. Cfr. A. SмITH, Theory of cit., I.i.3.8.

56. Cfr. Ibidem.

57. Cfr. Ibidem, I.i.1.10.

58. Cfr. Ibidem, I.i.3.8. 
amplía empíricamente las bases que suscitan la simpatía y la aprobación moral implícita, la cual puede ser conocida por un público amplio $^{59}$, lo que le permite una explicación quizá más realista del proceso de la simpatía.

Con todo, Smith no abandona la estructura de fondo del proceso humeano de la simpatía, consistente en una asociación de ideas e impresiones en la imaginación. Aunque no explica la cadena de asociaciones en la mente que Hume expone claramente, quizá para no salirse de su propósito introduciendo detalles innecesarios; podría perfectamente haberlo hecho, explicando las funciones de una imaginación ampliada, por medio de la asociación entre ideas e impresiones ${ }^{60}$. Algunos elementos dispersos en Theory of Moral Sentiments, que exponemos a continuación, nos remiten a la explicación que hace Hume del proceso de la simpatía.

Como no tenemos experiencia inmediata de lo que los otros sienten, afirma Smith, nos formamos una idea de la manera en que han sido afectados concibiendo lo que nosotros sentiríamos en la misma situación, copiando las impresiones de nuestros sentidos en nuestra imaginación. De este modo nos ponemos a nosotros mismos en su situación, llegando a ser en cierto modo una misma persona con el otro, formando así una idea de sus sensaciones, y sintiendo algo semejante a lo que siente, pero más débil. Concebir o imaginar que nosotros estamos, por ejemplo, en el mismo tormento, provoca un cierto grado de la misma emoción que el otro siente, en proporción a la vivacidad u opacidad de nuestra concepción ${ }^{61}$. Encontramos en esta explicación el mismo asociacionismo entre ideas e impresiones en la imaginación establecido por Hume. De la impresión en la imaginación de la situación en que se encuentra el otro surge una idea, la cual, en proporción a su vivacidad provoca, a la vez, una emoción o sentimiento que constituye la simpatía. La diferencia con Hume es que la idea no surge de mirar la expresión del otro, sino de ponerse con la imaginación en su situación.

59. Cfr. K. Haakonssen, The Science cit., 47.

60. Cfr. Ibidem, 48.

61. Cfr. A. SмITH, Theory of cit., I.i.1.2. 
Ese sentimiento de simpatía o de lo contrario, es la medida del grado de afección que debería tener el otro ante esa determinada situación. Lo explica Smith a través de su concepción del propio yo, que es idéntica a la de Hume. Cuando adoptamos los tormentos del otro, dice, los hacemos propios, y por eso nos afectan. Entonces, la afección correspondiente del espectador es la medida del grado de afección que debería tener la persona juzgada ante la misma situación ${ }^{62}$.

Pues bien, ¿por qué Reid considera que esta explicación se basa en una hipótesis? No lo explica cuando critica a Smith. Para responder a esta pregunta hay que referirse a explicaciones que encontramos en otras obras suyas. En Essays of Intellectual Powers considera que a partir de Descartes los filósofos han explicado la naturaleza a partir de hipótesis fundadas solo en probabilidades, por las que interpretan y unifican los fenómenos de una manera bella para la imaginación, constituyendo así un sistema filosófico ${ }^{63}$. Una de ellas, de la que Reid se ocupa especialmente, es la del Treatise de Hume, según la cual toda la actividad de la mente se reduce a unas percepciones originales, las impresiones y las ideas, y unas leyes de asociación entre ellas, a partir de las cuales se explica todo el mecanismo de los sentidos, la imaginación, la memoria, la creencia, las pasiones y la simpatía ${ }^{64}$. En la medida en que Smith ha adoptado la estructura de fondo del proceso humeano de la simpatía, su sistema puede ser calificado de hipotético por Reid.

Cualquier hipótesis respecto a las obras de Dios, dice Reid, tiene tan poca probabilidad como las conjeturas de un niño respecto a las obras de un hombre. La sabiduría de Dios excede la sabiduría del hombre más inteligente. Para conocerla hay que recurrir a la observación paciente de los fenómenos, como se ha hecho hasta ahora por los anatomistas en relación al cuerpo, y como se debe hacer en adelante respecto a la mente ${ }^{65}$.

62. Cfr. Ibidem I.i.1.2 y VII.ii.3.21.

63. Cfr. T. REID, Essays on Intellectual cit., 48.

64. Cfr. T. ReID, An Inquiry cit., 22. Las cursivas son mías.

65. Cfr. T. REID, Essays on Intellectual cit., 49. 


\section{LA SIMPATÍA REIDIANA SUPONE EL JUICIO DE UNA FACULTAD MORAL RACIONAL. MÉTOdo INDUCTIVO RESOLUTIVO}

A pesar de que Reid ha definido la simpatía como un instinto involuntario, no virtuoso en cuanto tal, afirma también la existencia de una simpatía virtuosa, que sigue, sin embargo, a una determinación de la voluntad, por ejemplo, a procurar alivio al afectado. Este tipo de simpatía supone y se subordina a una facultad moral, por la cual percibimos la bondad o maldad de las acciones a las que nos inclina dicha afección. Hay una diferencia importante entre la simpatía virtuosa en Smith, que constituye en sí misma una aprobación moral; y la simpatía virtuosa en Reid, que sigue la aprobación de una facultad racional, capaz de hacer juicios morales sobre las acciones.

Cuando Reid hace esta crítica, ya se había referido anteriormente, en su Inquiry a la existencia de facultades en la mente humana, cuya estructura depende de la sabiduría del arquitecto divino, y cuya realidad llega a ser conocida por nosotros por la observación y la experiencia de sus operaciones ${ }^{66}$. La realidad de la facultad moral, en concreto, es ampliamente desarrollada en Essays of Active Powers V, Chap. VII, una obra bastante posterior a la crítica de Reid a Smith, pero que, sin embargo, tiene sus raíces en una época temprana de la vida intelectual de Reid, como se puede ver en algunos manuscritos, y en sucesivas etapas de su carrera intelectual, dentro de la cual, una parte principal esta formada por sus clases en la Universidad de Glasgow ${ }^{67}$.

Afirmar la realidad metafísica de una facultad moral racional originaria - cuyo acto es una certeza moral o creencia, es decir un acto de la parte cogitativa, y no de la parte sensitiva de nuestra naturaleza ${ }^{68}$ - , cuando comenzaba a predominar la idea de que la aprobación moral es un sentimiento, producto de una asociación de ideas e impresiones en la mente, no es un asunto menor. Explicando cómo podemos aplicar nuestras nociones de bien y mal a nuestras acciones particulares, Reid critica a quienes piensan que nuestros

66. Cfr. An Inquiry cit., 11-12.

67. Cfr. T. REID, Essays on the Active cit., ix-xi.

68. Cfr. T. REID, Essays on Active cit., 345. 
sentimientos morales son apropiados para dicho propósito, sin suponer una facultad anterior capaz de juzgar de esos mismos sentimientos ${ }^{69}$. Seguramente Reid se refería a Hume y a Smith cuando hace estas consideraciones ${ }^{70}$. Tener simpatía por un carácter noble, dice Reid, es el efecto necesario de nuestro juicio previo acerca de su conducta ${ }^{71}$, y no al contrario. La esencia de la virtud, afirma en otro lugar, objeto de aprobación moral, no consiste en simpatizar con las pasiones y afecciones de los otros, ni en armonizar nuestra propia conducta con el tono de las pasiones de los otros, sino en vivir de acuerdo a nuestra propia conciencia, es decir a nuestra facultad moral, por la que conocemos nuestro deber ${ }^{72}$.

Reid demuestra la existencia de la facultad moral por el método inductivo newtoniano, que le parece accesible a todas las personas. Lo hace generalizando el método de análisis de Newton desde la observación de fenómenos físicos a la observación de fenómenos mentales observables físicamente, y desde esta última a la reflexión atenta sobre nuestros actos mentales. Su teoría de la percepción le lleva a establecer un puente entre la observación y la reflexión ${ }^{73}$. Así realiza la pretensión de Newton según la cual la filosofía moral, como parte de la filosofía natural, se perfeccionaría también por el mismo método ${ }^{74}$.

Así establece que el modo principal y directo de acceso a nuestras operaciones mentales es la reflexión atenta sobre ellas. A este modo le siguen dos que le están subordinados, de los cuales el primero es la atención a la estructura del lenguaje, y el segundo es una debida atención al curso de nuestras acciones y conducta ${ }^{75}$.

Pues bien, por el análisis de la estructura del lenguaje llegamos a la conclusión de que un sentimiento (feeling) no puede ser una aprobación moral ${ }^{76}$. Puede ser agradable, doloroso, o indife-

69. Cfr. Ibidem, 175.

70. Cfr. Ibidem, nota 94.

71. Cfr. Ibidem, 183.

72. Cfr. Ibidem, 300-301.

73. Cfr. Ellos, op. cit., 23-24.

74. Cfr. Newton, op. cit., 543.

75. Cfr. T. REID, Essays on Intellectual cit., 56.

76. Cfr. T. REID, Essays on Active cit., 346. 
rente. Puede ser débil o fuerte. Pero se expresa en el lenguaje por una sola palabra, o constituyendo el sujeto o el predicado dentro de un conjunto de palabras que conforman una proposición. El mero sentimiento no implica ni una afirmación ni una negación, por lo que no puede tener la cualidad de verdadero o falso, que distingue a las proposiciones de otras formas de expresarse, y a los juicios de otros actos de la mente. Afirmar, por ejemplo, que "yo tengo tal sentimiento", es, sin duda una proposición afirmativa, pero el sentimiento es en ella sólo un término de la proposición. En el juicio, nosotros podemos distinguir el objeto juzgado del acto de la mente por el cual juzgamos. En el mero sentimiento no es posible hacer esa distinción. El dolor de cabeza expresa un sentimiento, pero nadie puede decir que expresa un juicio.

De este modo, a través de la estructura del lenguaje, Reid llega a afirmar que no se puede realizar un juicio moral a partir de un sentimiento. Partiendo del segundo modo de acceso a nuestras operaciones mentales, es decir por la debida observación al curso de nuestras acciones y conducta, Reid afirma que cuando uno ve a un hombre ejercitándose en una causa noble y buena, uno siente agrado, afecto y estima por él; pero al mismo tiempo uno es consciente de que ese agrado, afecto y estima dependen enteramente del juicio que me he formado de su conducta. La aprobación de una buena acción es, por tanto, compleja, en la medida en que encontramos sentimiento, estima y un juicio. Frente a esa conducta puedo afirmar: "ese hombre obra bien y su conducta es altamente aprobable", o puedo decir: "la conducta de ese hombre me proporciona un sentimiento agradable". No existe ninguna regla de gramática o retórica, ningún uso del lenguaje, por los cuales esas dos frases pudieran tener el mismo significado. La primera expresa un juicio sobre la conducta de un hombre, y no dice nada sobre el que habla. La segunda testifica sólo lo concerniente al que habla, es decir, que tiene un sentimiento.

Reid se apoya en la evidencia universal del lenguaje para distinguir los juicios morales de los juicios acerca de la existencia de un sentimiento en un sujeto. Algunos lenguajes particulares, dice, pueden tener rarezas, o cosas absurdas introducidas por algún hombre 
eminente, por capricho o por juicios erróneos, seguidos por imitadores serviles. Pero, mientras haya hombres con entendimiento, no puede persistir el mismo absurdo en todas las lenguas, a través de todos los tiempos, a pesar de haber sido detectado.

El recurso metodológico a la estructura del lenguaje común y universal es empleado muchas veces por Reid, precisamente como un arma contra el sistema bipotético de Hume ${ }^{77}$, que, según los ejemplos anteriores, hace un mal uso del lenguaje ${ }^{78}$. Siguiendo la huella de su maestro Turnbull, para quien el lenguaje no ha sido inventado por los filósofos, sino ideado para expresar sentimientos comunes ${ }^{79}$, propios de la naturaleza, Reid afirma:

Sostengo que es cierto el principio general, según el cual cada distinción que encontramos en la estructura del lenguaje común es una distinción real, susceptible de ser percibida por el sentido común de la humanidad, que de hecho hace uso frecuentemente de él. Deseo que fuera más utilizado de lo que ha sido; porque creo que todo el sistema de la metafísica, o la gran parte de ella, puede educirse de él; y, junto con una cuidadosa reflexión sobre las operaciones de nuestra mente, no conozco nada que pueda dar más luz a las facultades humanas como una debida consideración de la estructura del lenguaje $\mathrm{e}^{80}$.

El sentido común de la humanidad no es, para Reid, un conjunto de opiniones más o menos mayoritarias sostenidas por los hombres en general, sino una facultad, como dice al comienzo de un manuscrito, Cura Prima, en el cual afirma que, a pesar de que el término "sentido común" se usa con frecuencia, no estamos inclinados a investigar su significado. Todos los hombres suponen que poseen esa facultad ${ }^{81}$.

77. Cfr. Ibidem, 345.

78. Cfr. A. Broadie, Reid in context cit., 44.

79. Cfr. G. Turnbull, The Principles of Moral and Christian Philosophy (Liberty Fund, Indianapolis, 2005)152.

80. Cfr. T. REID, The Correspondence of Thomas Reid (Edinburgh University Press, Edinburgh, 2002) 185.

81. Cfr. Cura Prima, en L. Marcil Lacoste, Claude Buffier and Thomas Reid, Two Common Sense Philosophers (McGill-Queen's University Press, Kingston, 1982) 181. 
Una facultad es un poder original y natural, que forma parte de la constitución de la mente ${ }^{82}$. El sentido común, así considerado, tiene un sentido específico, que puede ser investigado por el uso común del lenguaje. Constituye un grado de discernimiento y comprensión que se le atribuye sólo a personas de edad madura y no tiene defectos naturales. Es un talento, un regalo de la naturaleza, y, aunque puede ser mejorado, no se adquiere por educación, sino más bien esta última lo presupone. Con el término "sentido común" nos referimos a los poderes naturales del entendimiento que distinguen a los hombres de los brutos ${ }^{83}$.

Los principios racionales de la acción humana pertenecen al sentido común. Actuar razonablemente es una frase tan común como juzgar razonablemente, en todos los lenguajes ${ }^{84}$. Nosotros aprobamos inmediatamente la conducta de un hombre cuando se ve que tiene buenas razones para actuar así y, del mismo modo, la desaprobamos si pensamos que es contraria a la razón ${ }^{85}$. Una manera tan universal de hablar entre los hombres, común a los sabios y a los ignorantes en todas las naciones y en todas las lenguas, tiene que tener un significado. Suponer que son palabras sin significado, es tratar con desprecio al sentido común de la humanidad ${ }^{86}$.

Esto último es lo que ha hecho Hume, según Reid, al afirmar que "la razón es, y sólo debe ser, la esclava de las pasiones, no pudiendo pretender otro oficio que el de servirlas y obedecerlas" ${ }^{87}$. En esta misma línea, Smith afirma en Theory of Moral Sentiments que es absurdo e ininteligible suponer que los primeros principios de lo bueno y lo malo deriven de la razón, y no de un sentimiento inmediato $^{88}$. El problema es que, según Reid, para Hume, la razón juzga sólo en materias especulativas ${ }^{89}$. En efecto, dice Hume que la razón sólo consiste en el descubrimiento de la verdad o falsedad por

82. Cfr. T. REID, Essays on Intellectual cit., 25.

83. Cfr. Cura Prima cit., 181.

84. Cfr. T. REID, Essays on Active cit., 152.

85. Ibidem.

86. Ibidem.

87. D. Hume, Treatise cit., 415. Cfr. T. REID, Essays on Active cit., 352.

88. Cfr. A. SмITH, Theory of cit., VII.iii.2.7.

89. Cfr. T. REID, Essays on Intellectual cit., 352. 
medio de relaciones entre ideas o comprobación de hechos ${ }^{90}$. Por eso la razón es totalmente inerte, y nunca puede ser origen de un principio tan activo como lo es la conciencia o sentido moral. Esta afirmación explícita en Hume, se encuentra implícita en Smith, el cual, describe también la razón como una asociación de ideas, es decir como razón teórica, en History of Astronomy ${ }^{91}$.

El problema que late tras esa concepción, según Reid, es dar preeminencia a las ideas y a sus leyes de asociación en la imaginación, como si de ello dependiera el conocimiento. Para Reid, en cambio, conocemos la realidad y las facultades de las que depende el conocimiento, a partir de los juicios que realizamos. La primera operación de la mente no es la mera concepción de una cosa sin ninguna creencia acerca de ella, lo que Reid denomina simple aprehensión o idea, cuya comparación de unas con otras, percibiendo acuerdos y desacuerdos entre ellas, constituirían nuestros juicios o conocimiento ${ }^{92}$. Reid considera que conocemos, más bien, por medio de juicios naturales y originales, de cuyo análisis se derivan elementos separados que son las simples aprehensiones, las cuales no son mostradas separadamente por la naturaleza ${ }^{93}$.

Pues bien, nosotros conocemos nuestras facultades naturales, dice Reid, por medio de las operaciones que se realizan dentro de nosotros, de las que somos conscientes, y de sus signos en las mentes de los otros. Por medio del juicio último acerca de lo que es bueno, malo o indiferente en la conducta de los agentes morales, por la aprobación de la buena conducta y la desaprobación de la mala a causa de ese juicio, y por la emoción agradable que acompaña a la obediencia, y por la desagradable que acompaña a la desobediencia a sus dictados, conocemos nuestra facultad moral, llamada también conciencia o sentido moral ${ }^{94}$.

90. Cfr. D. Hume, Treatise cit., 457-458.

91. Cfr. A. Smith, Principles which lead cit., 33-53.

92. Cfr. An Inquiry cit., 29-30.

93. Cfr. Ibidem.

94. Cfr. T. REID, Essays on Active cit., 185. 
Esos juicios constituyen un campo de observación, que nos permite tener evidencia acerca de la existencia de nuestra facultad moral, como causa eficiente de ellos. En la filosofía natural, tanto física como moral, buscamos, por medio del método inductivo, las causas de los fenómenos observados. Ahora bien, en la física llegamos a establecer las causas de los fenómenos sin conocer la entidad de la causa, a la cual sólo conocemos como un fenómeno observable que antecede a un fenómeno consecuente. Por eso en la física no se busca el conocimiento de la causa eficiente de los fenómenos, sino sólo las leyes por medio de las cuales ellos son regulados ${ }^{95}$. No sabemos si un cuerpo se pone en movimiento por una actividad existente en sí mismo o por la eficiencia de una causa externa. Esa investigación sobre la entidad de las causas físicas pertenece a la Metafísica ${ }^{96}$.

En la filosofía moral, en cambio, que es parte también de la filosofía natural, la causalidad inteligente como causalidad eficiente en la mente, es cognoscible a través de sus efectos, como es el caso de la facultad moral, a cuya realidad llegamos a través de sus operaciones - es decir, el juicio último acerca de las acciones, el lenguaje y la conducta - como hemos visto. Aunque no podemos observarla, por ser una causalidad inteligente, podemos reflexionar sobre sus efectos, por lo que su realidad es tan filosóficamente fundada como la de las causas físicas. $\mathrm{Al}$ considerar la realidad de esta facultad, Reid respeta la norma newtoniana de que bypotheses non fingo $0^{97}$. "Nosotros no vemos los corazones de los hombres", dice Reid, "tampoco los principios por los cuales actúan; pero hay signos externos de sus principios y disposiciones, los cuales, aunque no incontestables, pueden a veces ser más confiables que sus profesiones"98.

\section{ConClusiones}

Al criticar el sistema de la simpatía de Smith, Reid plantea un problema filosófico antiguo, si la verdad o maldad de nuestras acciones

95. Cfr. T. REID, The Correspondence cit., 127.

96. Cfr. Ibidem.

97. Cfr. NeWTON, op. cit., 371.

98. Cfr. T. REID, Essays on Intellectual cit., 559. 
se encuentra al alcance de una facultad moral en el hombre. Sin embargo, la manera naturalista de enfrentar el problema es contemporánea. Desde el contexto ilustrado en que desarrolló su filosofía, en que predominaba la admiración por el método newtoniano, sobre todo por sus resultados en la física, supo privilegiar, con rigor, lo esencial a las reglas del filosofar de Newton, que es el método de análisis inductivo, según el cual se puede acceder al conocimiento de la existencia de realidades metafísicas, como son las facultades, aunque no se las pueda observar, salvando así la veracidad de los juicios morales. Estos últimos no dependen de una razón constructiva, a partir de la asociación de ideas e impresiones en la mente, sino de una constitución natural, que permite el acceso a la verdad, observable a partir de sus efectos. La razón constructiva, en cambio, se explica a partir de una hipótesis acerca de los elementos simples de nuestra mente, a partir de los cuales, y por medio de mecanismos más o menos complejos, se irían construyendo juicios morales. 